OPEN ACCESS

Edited by:

Alessio Mazzoni,

University of Florence, Italy

Reviewed by:

Roberta Amoriello,

University of Florence, Italy

Pieter Meysman,

University of Antwerp, Belgium

*Correspondence:

Jianhua Xu

jhxu1976@gzucm.edu.cn

Kejian Wang

kejian-wang@foxmail.com

Dawei Wang

david@gzucm.edu.cn

${ }^{\dagger}$ These authors have contributed equally to this work

Specialty section:

This article was submitted to

T Cell Biology,

a section of the journal

Frontiers in Immunology

Received: 02 September 2021

Accepted: 24 December 2021

Published: 27 January 2022

Citation:

Li Y, Hu J, Wang Y, Liu D, Shi Y,

Zhang J, Liu Y, Lin D, Lin J, Hu W,

He H, Wang W, Fan W, Li L, Wang D,

Wang K and XU J (2022) T-Cell

Repertoire Characteristics of

Asymptomatic and Re-Detectable

Positive COVID-19 Patients.

Front. Immunol. 12:769442.

doi: 10.3389/fimmu.2021.769442

\section{T-Cell Repertoire Characteristics of Asymptomatic and Re-Detectable Positive COVID-19 Patients}

\author{
Yizhe $\mathrm{Li}^{1 \dagger}$, Jian $\mathrm{Hu}^{2 \dagger}$, Yongsi Wang ${ }^{3 \dagger}$, Dongdong $\mathrm{Liu}^{2+}$, Yaling Shi ${ }^{4}$, Jiaqi Zhang ${ }^{1,2}$, \\ Yuntao Liu ${ }^{5}$, Dongzi Lin ${ }^{6}$, Jing Lin ${ }^{7}$, Wei Hu ${ }^{1}$, Haolan He ${ }^{8}$, Wei Wang ${ }^{6}$, Wentao Fan ${ }^{3}$, \\ Linlin $L^{3}{ }^{3}$, Dawei Wang ${ }^{9 *}$, Kejian Wang ${ }^{10,11^{*}}$ and Jianhua $X u^{1 *}$ \\ 1 Department of Laboratory Medicine, Shunde Hospital of Guangzhou University of Chinese Medicine, Foshan, China, \\ 2 Department of Laboratory Medicine, The Second Affiliated Hospital of Guangzhou University of Chinese Medicine, \\ Guangzhou, China, ${ }^{3}$ Department of Translational Medicine Research Institute, Guangzhou Huayin Medical Laboratory Center \\ Ltd., Guangzhou, China, ${ }^{4}$ Department of Laboratory Medicine, Guangzhou Eighth People's Hospital, Guangzhou Medical \\ University, Guangzhou, China, ${ }^{5}$ Emergency Department, The Second Affiliated Hospital of Guangzhou University of Chinese \\ Medicine, Guangzhou, China, ${ }^{6}$ Department of Laboratory Medicine, The Fourth People's Hospital of Foshan, Foshan, China, \\ ${ }^{7}$ Department of Clinical Laboratory, The First People's Hospital of Foshan, Foshan, China, ${ }^{8}$ Department of Infectious \\ Diseases, Guangzhou Eighth People's Hospital, Guangzhou Medical University, Guangzhou, China, ${ }^{9}$ Department of \\ Pulmonary and Critical Care Medicine, Shunde Hospital of Guangzhou University of Chinese Medicine, Foshan, China, ${ }^{10}$ The \\ Third Affiliated Hospital of Shandong First Medical University (Affiliated Hospital of Shandong Academy of Medical Sciences), \\ Jinan, China, ${ }^{11}$ Innovative Institute of Chinese Medicine and Pharmacy, Shandong University of Traditional Chinese \\ Medicine, Jinan, China
}

The prevention of the COVID-19 pandemic is highly complicated by the prevalence of asymptomatic and recurrent infection. Many previous immunological studies have focused on symptomatic and convalescent patients, while the immune responses in asymptomatic patients and re-detectable positive cases remain unclear. Here we comprehensively analyzed the peripheral T-cell receptor (TCR) repertoire of 54 COVID19 patients in different courses, including asymptomatic, symptomatic, convalescent, and re-detectable positive cases. We identified a set of $\mathrm{V}-\mathrm{J}$ gene combinations characterizing the upward immune responses through asymptomatic and symptomatic courses. Furthermore, some of these $\mathrm{V}-\mathrm{J}$ combinations could be awakened in the re-detectable positive cases, which may help predict the risk of recurrent infection. Therefore, TCR repertoire examination has the potential to strengthen the clinical surveillance and the immunotherapy development for COVID-19.

Keywords: COVID-19, T-cell receptor, asymptomatic patient, re-detectable positive case, immune repertoire sequencing

\section{INTRODUCTION}

As a highly infectious virus, the severe acute respiratory syndrome coronavirus 2 (SARS-CoV-2) caused the pandemic of coronavirus disease 2019 (COVID-19) $(1,2)$. The clinical manifestations of infected patients ranged from asymptomatic condition to severe symptoms (3). Moreover, among the COVID-19 convalescent patients, some tested positive again after discharge (i.e., re-detectable positive) (4). 
The global efforts to end COVID-19 are complicated by the prevalence of asymptomatic and recurrent infection. Many previous immunological studies have focused on symptomatic and convalescent patients (5-12), while the immune responses in asymptomatic patients and re-detectable positive cases remain unclear $(13,14)$. Unlike symptomatic patients who can be effectively identified by clinical features, asymptomatic carriers may inadvertently transmit virus to close contacts and reshape the dynamics of infection in population $(15,16)$. Although most re-detectable positive cases have minor symptoms and hardly disease progression upon readmission, their potential infectivity and immunological characterization remain undefined $(17,18)$. We performed a comprehensive analysis of the transcriptomic profiles of PBMCs from COVID-19 patients in our previous studies (19). However, the pathogenesis is not fully understood at present.

The antiviral adaptive immunity is greatly dependent on the activation of T-cells, which can selectively eliminate virusinfected host cells (20). The specificity toward viral antigens is determined by the structure of the T-cell receptor (TCR) repertoire (21). Based on the advances in sequencing technologies, the biased TCR repertoire in various infectious diseases has been revealed (22-24). Kuri-Cervantes et al. demonstrated the different trajectories of the immunologic state in moderate, severe, and recovered COVID-19 patients (25). Wauters et al. provided deep-immune trajectories of mild to critical COVID-19 by analyzing bronchoalveolar lavage samples (26). Thus, an in-depth study on the TCR characteristics in different courses of COVID-19 is critically needed (27-31).

Here we analyzed the peripheral TCR repertoire of 54 COVID19 patients in different courses (including asymptomatic, symptomatic, convalescent, and re-detectable positive cases) along with 16 healthy donors. In particular, our results presented the unique immunological features of asymptomatic patients and re-detectable positive cases, which could provide help for clinical management and therapy development.

\section{RESULTS}

\section{Demographic and Clinical Characteristics}

A total of 54 patients with laboratory-confirmed COVID-19 disease were enrolled, including 11 asymptomatic (ASY), 19 symptomatic (SYM), 14 convalescent (CON), and 10 redetectable positive (RDP) cases. Moreover, 16 healthy donors (HD) were recruited as the control group. As shown in Table 1, no significant difference in age or sex was identified between the HD group and any of the patient groups (as measured by the two-tailed Mann-Whitney U-test or Fisher's exact test). Except for one patient with preexisting chronic pharyngitis, most ASY cases had no obvious clinical symptoms during the whole disease course. All HD subjects and most ASY patients had no underlying comorbidity, while some of the SYM, CON, and RDP patients were diagnosed with hypertension, hyperlipidemia, diabetes mellitus, cardiovascular disease, chronic liver disease, or chronic kidney disease (32). The routine laboratory test results (Supplementary Table 1) showed that the lymphocyte levels in the SYM and CON groups were significantly lower than that in the HD group (two-tailed Mann-Whitney U-test $\mathrm{p}<0.05$ ). However, such difference was not observed in the ASY or RDP group.

Since HLA molecules play a crucial role in shaping the TCR repertoire (33), we also sought to determine whether the frequency of a certain HLA allele was imbalanced across groups. By performing Fisher's exact test following a previously published procedure (22), we confirmed that none of the HLA alleles showed significant difference (Figure 1 and Supplementary Table 2), which consolidated our following findings in the TCR repertoire.

\section{Patients in Different Courses Have Heterogeneous TCR Characteristics}

On average, 10.6 million clean reads were obtained from each sample in sequencing (ranging from 6.2 to 13.2 million, see Methods). No significant difference was found between groups in number of reads, clonotypes, or $\mathrm{V}-\mathrm{J}$ genes (Figures 2A-C). No significant difference in clonal diversity (as measured by D50 index and Shannon entropy) was detected between any two groups (Figures 2D, E), suggesting that COVID-19 might not necessarily induce a widespread change in TCR repertoire diversity. Also, we analyzed the differential expression of amino acid clonotypes in patients and healthy donors (Figure 3). The clonotype AAPVFVLGLQAVSTDTQY was significantly decreased in both SYM and ASY groups. Moreover, the clonotype EGAGLLQYPPLSKLF showed a significantly lower expression in both CON and RDP groups.

In the context of $\mathrm{V}-\mathrm{J}$ gene usage, the similarity between subjects was measured by the Spearman's correlation (i.e., stronger positive correlation indicates higher similarity) following a previously published procedure (34). Taking HD as a reference, the highest discrepancy was found between SYM and HD. On the other hand, ASY showed significantly higher similarity to HD (Figure $\mathbf{4 A}$ ), suggesting that asymptomatic patients may be subject to less changes in cellular immunology.

Furthermore, taking RDP as a reference, ASY showed the highest similarity as compared to other patient groups (Figure 4B). This finding is consistent with the fact that most RDP patients, just as ASY ones, exhibited no obvious clinical symptoms.

\section{Dynamics of Gene Usage in Different Courses of COVID-19}

We further analyzed the dynamics of specific V-J gene expression to identify potential key factors associated with different courses of COVID-19. Using the expression level in $\mathrm{HD}$ as a baseline, we identified $73 \mathrm{~V}-\mathrm{J}$ pairings that exhibited not only alteration in ASY, but even more drastic changes in SYM (Cuzick's test for monotonic trend $\mathrm{p}<0.05$, Figure 5A and Supplementary Table 3) (35). Of note, a significant portion $\left(30.1 \%\right.$, hypergeometric test $\left.\mathrm{p}=1.08 \times 10^{-13}\right)$ of the monotonic $\mathrm{V}-\mathrm{J}$ pairings also showed differential usage in RDP course (RDP 
TABLE 1 | Baseline characteristics of study subjects.

\begin{tabular}{|c|c|c|c|c|c|}
\hline & $\begin{array}{l}\text { Healthy } \\
(n=16)\end{array}$ & $\begin{array}{l}\text { Asymptomatic } \\
\qquad(n=11)\end{array}$ & $\begin{array}{l}\text { Symptomatic } \\
\qquad(n=19)\end{array}$ & $\begin{array}{l}\text { Convalescent } \\
\qquad(n=14)\end{array}$ & $\begin{array}{l}\text { Re-detectable positive } \\
\qquad(n=10)\end{array}$ \\
\hline \multicolumn{6}{|l|}{ Severity of the first diagnosis (n, \%) } \\
\hline Asymptomatic & 0 & $11(100 \%)$ & 0 & 2 (14.29\%) & $1(10 \%)$ \\
\hline Mild & 0 & 0 & $2(10.53 \%)$ & $2(14.29 \%)$ & $1(10 \%)$ \\
\hline Moderate & 0 & 0 & $16(84.21 \%)$ & $4(28.57 \%)$ & $8(80 \%)$ \\
\hline Severe & 0 & 0 & $1(5.26 \%)$ & $6(42.86 \%)$ & 0 \\
\hline Age (years, median and IQR) & $34.50(27.25-44.25)$ & $33.00(21.00-44.00)$ & $32.00(23.00-48.00)$ & $46.50(23.50-65.75)$ & $30.50(24.25-59.75)$ \\
\hline Male (n, \%) & $9(56.25 \%)$ & $4(36.36 \%)$ & $12(63.16 \%)$ & $10(71.43 \%)$ & $4(40 \%)$ \\
\hline Female (n, \%) & $7(43.75 \%)$ & 7 (63.64\%) & 7 (36.84\%) & $4(28.57 \%)$ & $6(60 \%)$ \\
\hline \multicolumn{6}{|l|}{ Comorbidities (n, \%) } \\
\hline Hypertension & 0 & 0 & $2(10.53 \%)$ & $1(7.14 \%)$ & $2(20 \%)$ \\
\hline Hyperlipidemia & 0 & 1 (9.09\%) & $2(10.53 \%)$ & 0 & 0 \\
\hline Diabetes & 0 & 0 & $1(5.26 \%)$ & $1(7.14 \%)$ & $1(10 \%)$ \\
\hline Cardiovascular disease & 0 & 0 & 0 & $1(7.14 \%)$ & 0 \\
\hline Chronic liver disease & 0 & 0 & $2(10.53 \%)$ & $1(7.14 \%)$ & $1(10 \%)$ \\
\hline Chronic kidney disease & 0 & 0 & 0 & $1(7.14 \%)$ & 0 \\
\hline \multicolumn{6}{|l|}{ Signs and symptoms (n, \%) } \\
\hline Cough & 0 & 0 & $13(68.42 \%)$ & $4(28.57 \%)$ & $1(10 \%)$ \\
\hline Expectoration & 0 & 0 & $11(57.89 \%)$ & $4(28.57 \%)$ & $1(10 \%)$ \\
\hline Rhinorrhoea & 0 & 0 & 2 (10.53\%) & 0 & 0 \\
\hline Headache & 0 & 0 & $6(31.58 \%)$ & 0 & 0 \\
\hline Fatigue & 0 & 0 & 7 (36.84\%) & 0 & 0 \\
\hline Sore throat & 0 & 0 & $3(15.79 \%)$ & 0 & $1(10 \%)$ \\
\hline Diarrhea & 0 & 0 & 0 & $1(7.14 \%)$ & 0 \\
\hline Chest tightness & 0 & 0 & $3(15.79 \%)$ & 2 (14.29\%) & 0 \\
\hline Myalgia & 0 & 0 & $3(15.79 \%)$ & $1(7.14 \%)$ & 0 \\
\hline Chills & 0 & 0 & $4(21.05 \%)$ & $1(7.14 \%)$ & $1(10 \%)$ \\
\hline Shortness of breath & 0 & 0 & $1(5.26 \%)$ & $1(7.14 \%)$ & 0 \\
\hline Nausea and vomiting & 0 & 0 & $4(21.05 \%)$ & 0 & 0 \\
\hline Dry throat & 0 & $1(9.09 \%)$ & $3(15.79 \%)$ & 0 & $1(10 \%)$ \\
\hline Gastrointestinal reaction & 0 & 0 & $1(5.26 \%)$ & 0 & $1(10 \%)$ \\
\hline Palpitation & 0 & 0 & 0 & 0 & $1(10 \%)$ \\
\hline Abdominal distension, nausea and retching & 0 & 0 & $2(10.53 \%)$ & 0 & 0 \\
\hline Poor stomach intake & 0 & 0 & $1(5.26 \%)$ & 0 & $1(10 \%)$ \\
\hline Erythra & 0 & 0 & $1(5.26 \%)$ & 0 & 0 \\
\hline Rhinobyon & 0 & 0 & 0 & 0 & 0 \\
\hline
\end{tabular}

vs. HD, Mann-Whitney $U$ test $p<0.05$, Figure 5B and Supplementary Table 4). The dynamics of these feature V-J pairings indicated that a certain immunological memory for SARS-CoV-2 could be awakened upon recurrent infection.

\section{DISCUSSION}

Asymptomatic and recurrent infection further increased the difficulty of COVID-19 prevention. Here we presented an immunological landscape of COVID-19 patients in different courses by performing TCR repertoire sequencing. In particular, while a series of prior studies focused on the immunological profiles of moderate, severe, or recovered patients, our results provided insights into the characteristics of ASY and RDP patients.

Despite numerous recent studies on COVID-19 patients, our analysis adds understanding of the immune responses to SARSCoV-2 in several aspects. First of all, it has been repeatedly reported that symptomatic patients exhibit more severe clinical manifestations than asymptomatic ones $(13,36-38)$. In agreement with such observation, we found that the usage of certain $\mathrm{V}$ and $\mathrm{J}$ gene segments exhibited a monotonic trend through HD, ASY, and SYM groups, indicating potential upward immune responses from asymptomatic to symptomatic course. The V-J combinations showing monotonic changes can help narrow down the search scope of TCR specific to SARS-CoV-2 antigens, which may facilitate the development of highly targeted immunotherapies, such as engineered T-cells for infusion $(39,40)$.

Next, the risk factors of recurrent infection are not yet well defined (14), which urges development of useful biomarkers to enhance clinical surveillance. We found that a proportion of $\mathrm{V}-\mathrm{J}$ combinations evoked by asymptomatic and symptomatic infection also changed in re-detectable positive cases. Such observation indicated the possibility of examining immune repertoire as a stringent criterion of complete recovery and an early predictor of high-risk individuals prone to recurrent infection.

Several limitations of our study should be taken into consideration. Firstly, four COVID-19 courses were characterized by blood samples collected from different individuals, rather than sequential samples from the same set of patients. Therefore, additional longitudinal studies are needed to calibrate the immunological trajectories of COVID-19. Secondly, we enrolled a limited number of patients, which makes it difficult to account for 
A
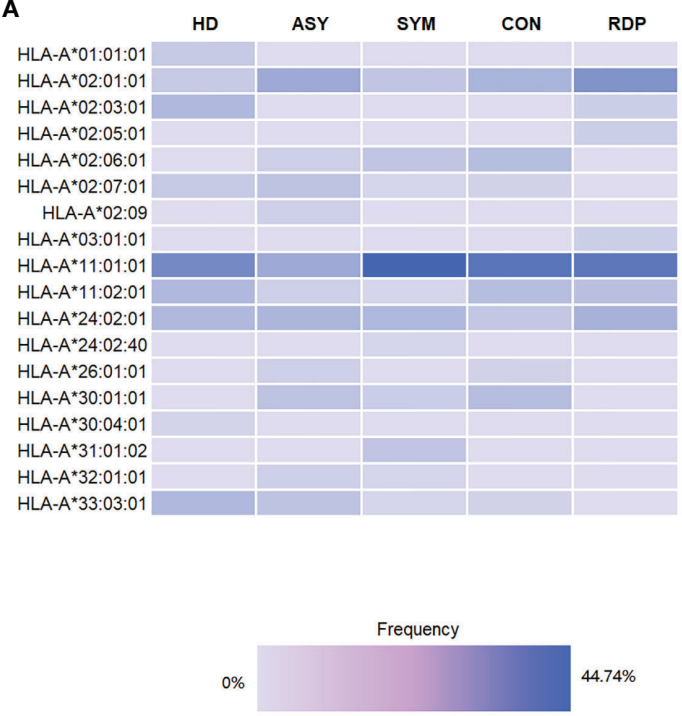

B

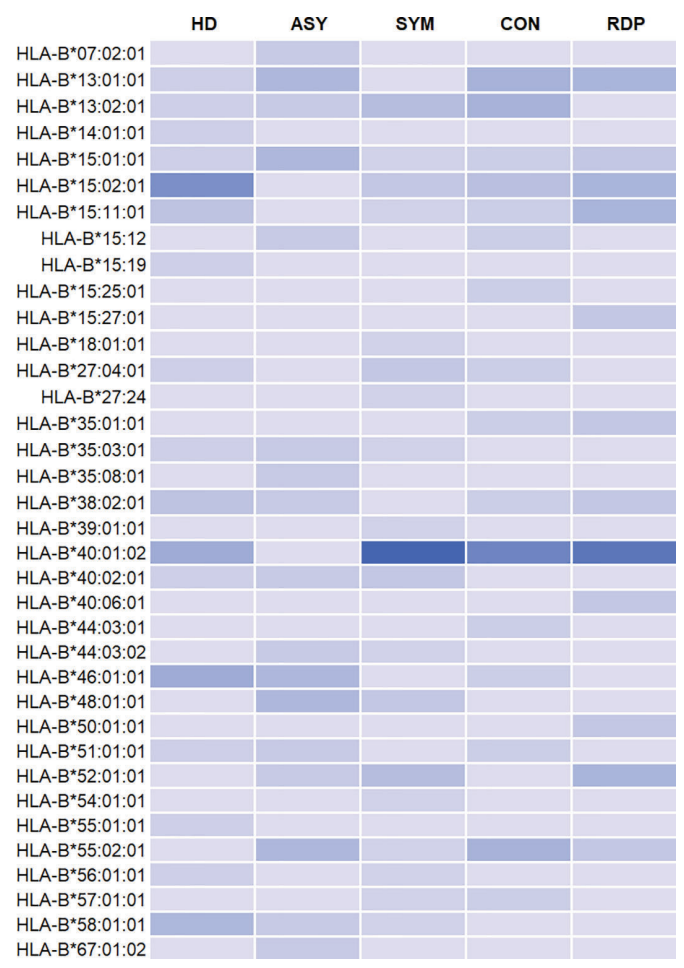

factors (e.g., diverse therapies and certain comorbidities) that may have divergent immunomodulatory effects. Subsequent efforts will optimally require larger patient cohorts to control for variations in treatment protocol and prevalence of comorbidities.

Altogether, this study provided novel insights into the TCR profiles of COVID-19 patients in different courses. A better understanding of the adaptive immunity in COVID-19 could strengthen clinical surveillance and immunotherapy development. Further large cohort studies on immune repertoire $(41,42)$ would be required to extend our findings.

\section{METHODS}

\section{Patients}

This study was approved by the ethics committees of the above four participant hospitals. Written informed consent was obtained from all participants enrolled in this study. Between March and May in 2020, a total of 54 patients with laboratory-confirmed COVID-19 disease were enrolled at the Guangzhou Eighth People's Hospital, the Shunde Hospital of Guangzhou University of Chinese Medicine, the Fourth People's Hospital of Foshan, and the First People's Hospital of Foshan, China. The COVID-19 patients were classified into four groups: asymptomatic group, symptomatic group, convalescent group, and re-detectable positive group.

According to the Protocol for Prevention and Control of COVID-19 (Edition 6) of National Health Commission of
China (43), the asymptomatic group were those who had a positive COVID-19 RT-PCR test or specific serum IgM antibodies, diagnosed as COVID-19, but without self-perception or clinically recognizable symptoms during the whole disease course. The symptomatic group had evident clinical symptoms and were diagnosed as COVID-19 according to the Diagnosis and Treatment Protocol for Novel Coronavirus Pneumonia (Version 7) of National Health Commission of China (44). In the convalescent group, patients were those with body temperature that returned to normal for more than 3 days, respiratory symptoms significantly improved, inflammation on pulmonary obviously absorbed, and nucleic acid tests of respiratory tract samples proved negative for two consecutive times (sampling interval being at least $24 \mathrm{~h}$ ). The re-detectable positive group was defined as patients with re-positive results of SARS-CoV-2 nucleic acid during the follow-up period after discharge from the hospital. The time between the re-positive results of the PCR test and the discharge time from the hospital ranged from 2 weeks to 1 month. Besides, 16 health donors were all enrolled at Shunde Hospital of Guangzhou University of Chinese Medicine, China, who tested negative for SARS-CoV-2 and exhibited no respiratory symptoms. Patient demographics and clinical manifestations were retrospectively reviewed. All patients had routine laboratory investigations, including complete blood count, liver function tests, blood gases analysis, and coagulation tests.

According to the Diagnosis and Treatment Protocol for Novel Coronavirus Pneumonia (Version 7) of China, the severity of 


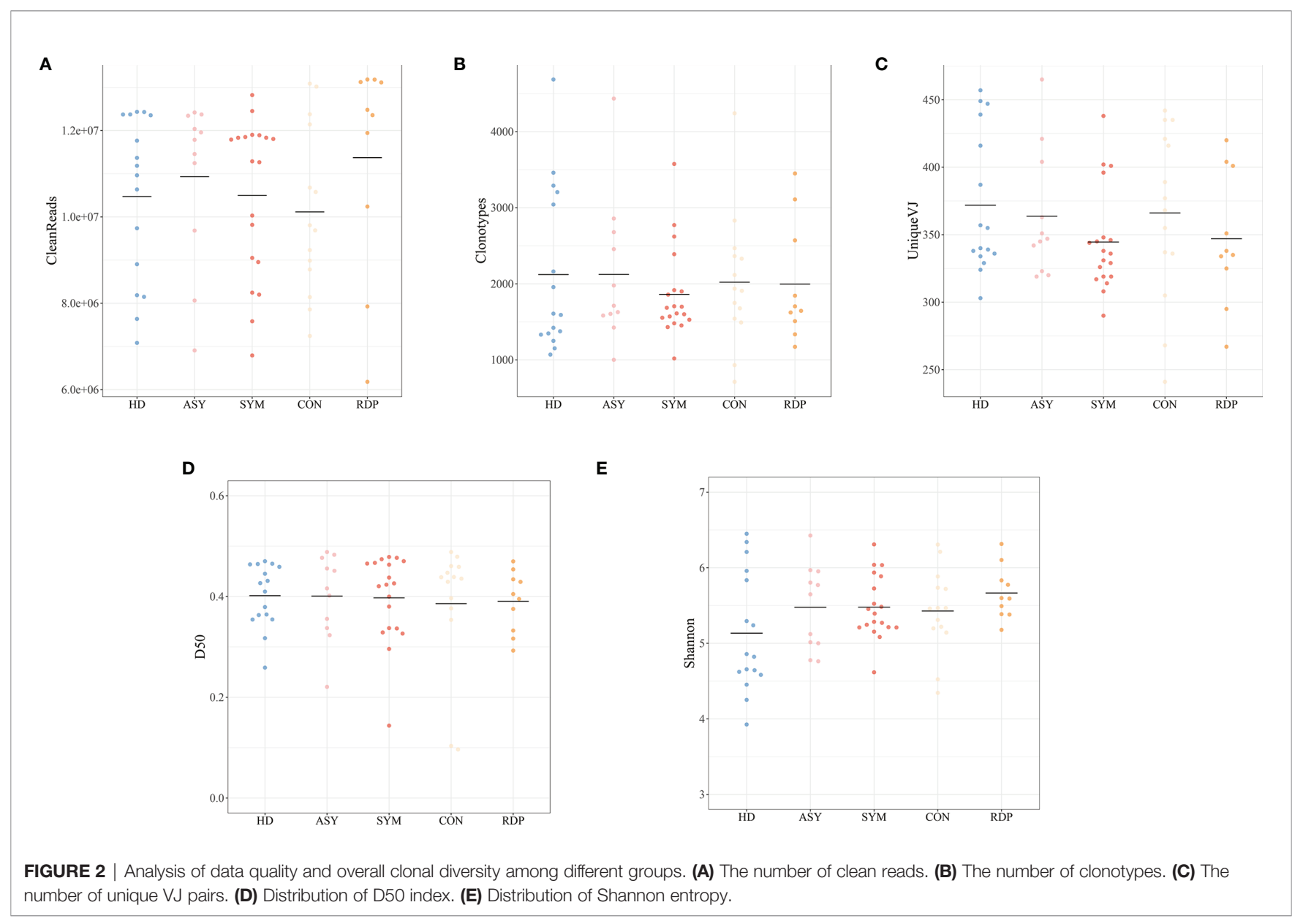

disease was as follows: (1) mild cases displayed clinical symptoms with no imaging manifestations of pneumonia; (2) moderate cases were characterized by fever and respiratory symptoms with radiological manifestations of pneumonia; (3) severe cases must meet one of the following criteria: respiratory distress $(\geq 30$ breaths/min), oxygen saturation $\leq 93 \%$ at rest, or arterial partial pressure of oxygen $(\mathrm{PaO} 2) /$ fraction of inspired oxygen $(\mathrm{FiO} 2) \leq 300 \mathrm{mmHg}(1 \mathrm{mmHg}=0.133 \mathrm{kPa})$.

\section{RNA Extraction and cDNA Synthesis}

Peripheral venous blood was collected and placed into the vacutainer tube. The time points of sample collection are shown in Supplementary Table 5. Peripheral blood mononuclear cells (PBMCs) are isolated from 2 4 ml human peripheral blood by Ficoll-Paque density gradient. Total RNA was isolated from PBMCs using TRIzol reagent (Invitrogen, Carlsbad, California, USA) according to the manufacturer's instruction (miRNeasy Mini Kit, Qiagen, Germany). The RNA quality inspection adopts Agilent 2100, and the quality control standard is RIN > 7.0, $28 \mathrm{~S} / 18 \mathrm{~S} \geq 1.0$.

TCR cDNA libraries for high-throughput sequencing were prepared by $5^{\prime}$ rapid amplification of cDNA ends (RACE) using the SMARTScribe ${ }^{\text {TM }}$ Reverse Transcriptase (Clontech, Mountain View, California, USA) as previously described $(45,46)$.
Briefly, $0.6 \mu \mathrm{g}$ of total RNA was mixed with the primer BC1R (Supplementary Table 6), which is specific for human TCR $\beta$ cDNA synthesis. To denature RNA and anneal the priming oligonucleotides, RNA was incubated at $70^{\circ} \mathrm{C}$ for $2 \mathrm{~min}$ and then at $42^{\circ} \mathrm{C}$ for $3 \mathrm{~min}$. Switch_oligo and SMARTScribe reverse transcriptases were added for $25 \mu \mathrm{l}$ template switching and the cDNA synthesis reaction, which was performed at $42^{\circ} \mathrm{C}$ for 60 min. 5U uracil DNA glycosylase (UDG) was added for digestion at $37^{\circ} \mathrm{C}$ for $40 \mathrm{~min}$, and the product was purified with MinElute PCR Purification Kit (Qiagen, Germany).

\section{TCR Library Preparation}

Two-round PCR was performed for TCR library preparation. For the first round of PCR amplification, $45 \mu \mathrm{l}$ of cDNA from the synthesis reaction was mixed with primers and Q $5^{\circledR}$ HighFidelity 2X Master Mix (NEB, USA). The PCR program began with an initial denaturation at $95^{\circ} \mathrm{C}$ for $1.5 \mathrm{~min}$, followed by 18 cycles of denaturation at $95^{\circ} \mathrm{C}$ for $10 \mathrm{~s}$, annealing of primer to DNA at $60^{\circ} \mathrm{C}$ for $20 \mathrm{~s}$, and extension at $72^{\circ} \mathrm{C}$ for $40 \mathrm{~s}$, and ended with an extension at $72^{\circ} \mathrm{C}$ for $4 \mathrm{~min}$. For the second round of PCR amplification, the product from the first round of PCR was purified by QIAquick PCR Purification Kit (Qiagen, Germany), and $10 \mu \mathrm{l}$ of the purified product was used in each $25-\mu \mathrm{l}$ PCR reaction. The reaction was performed for 14 cycles using the 
A

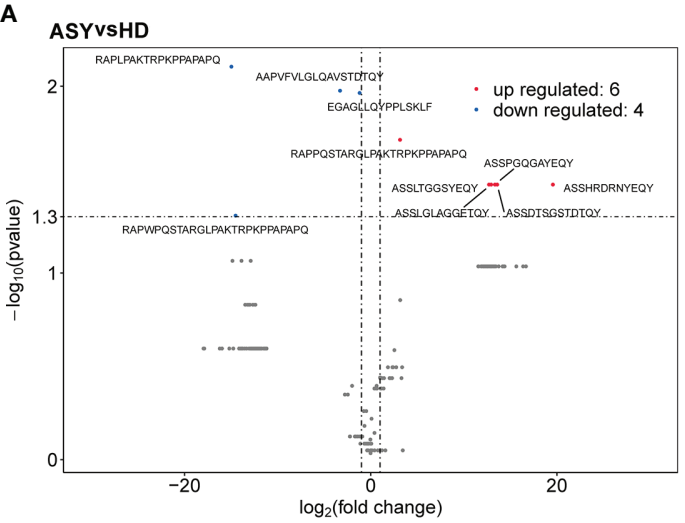

C

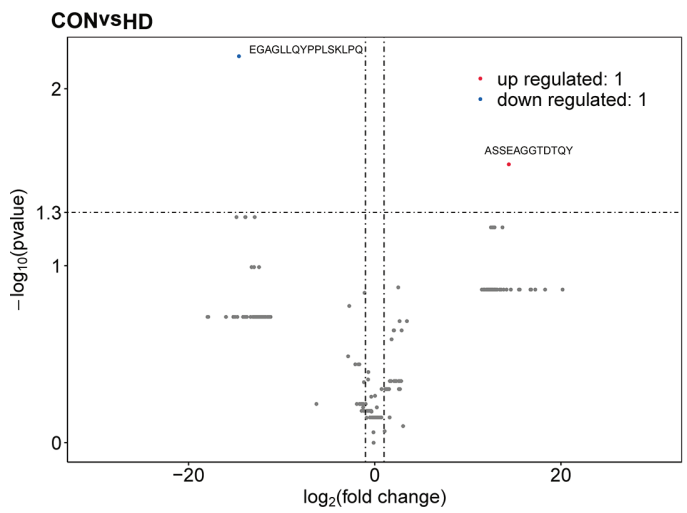

B

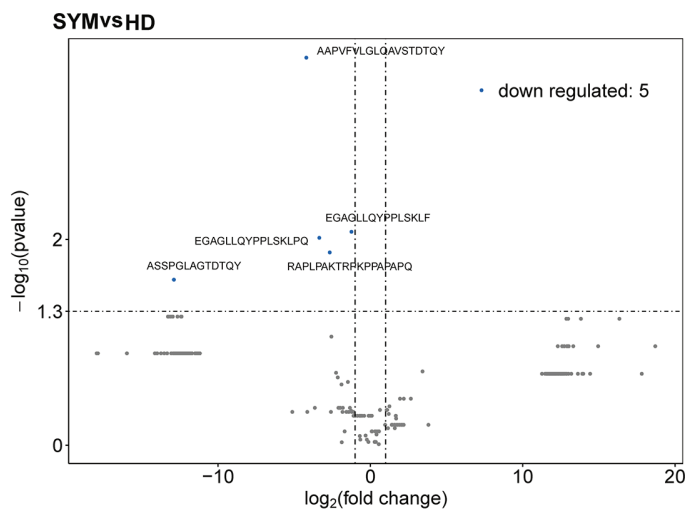

D

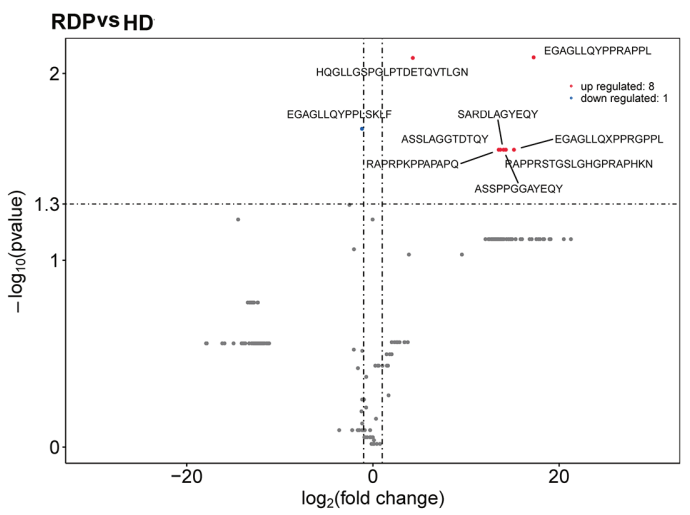

FIGURE 3 | Volcano plots showing differential expression (i.e., $|\log 2(F C)|>1, p<0.05$ ) of amino acid clonotypes between patients and healthy donors. (A) ASY vs. HD. (B) SYM vs. HD. (C) CON vs. HD. (D) RDP vs. HD.

A

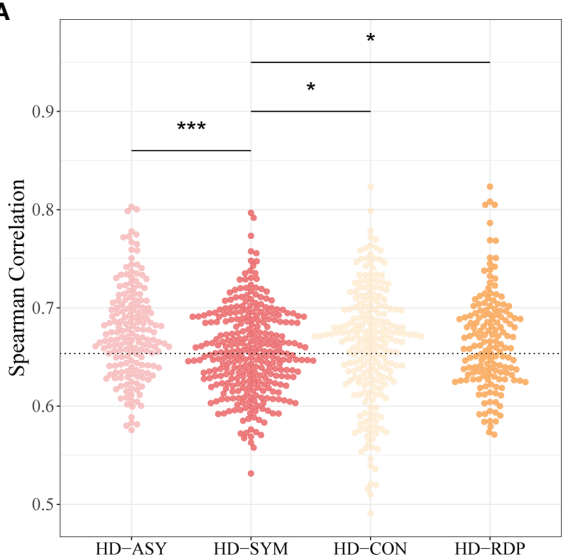

B

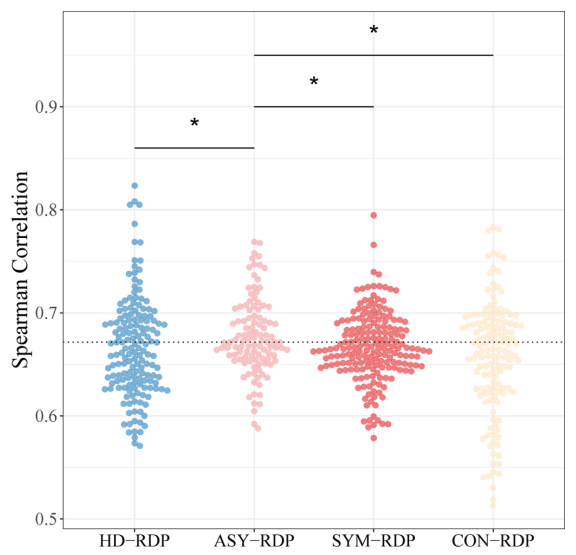

FIGURE 4 | Spearman's correlation coefficients of V-J combination profiles between different groups. (A) The HD-ASY similarity is significantly higher than that of HD-SYM. (B) ASY showed the highest similarity to RDP among all groups. Statistical significance of the difference between groups is denoted as * for $P<0.05$, *** for $\mathrm{P}<0.001$. 
A

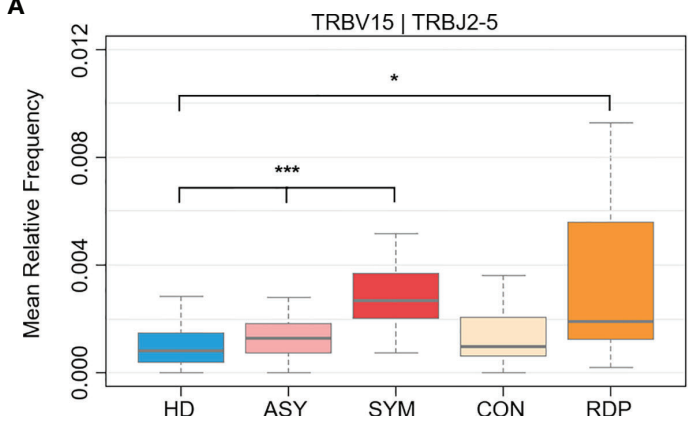

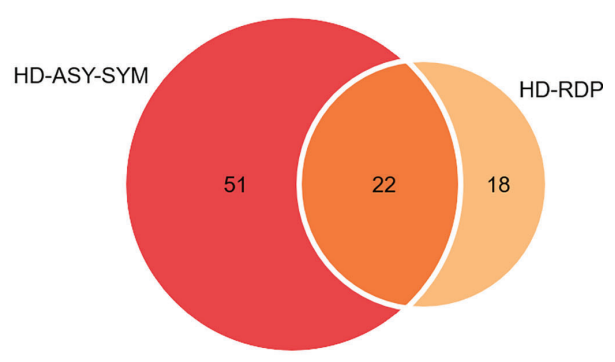

FIGURE 5 | Differential V-J gene usage between different groups. (A) An exemplary V-J combination showing a monotonic increase through HD, ASY, and SYM courses, as well as a significant elevation in RDP group. (B) Among the monotonic $\mathrm{V}-\mathrm{J}$ combinations, many were also differentially expressed in RDP cases. Statistical significance of the difference between groups is denoted as * for $\mathrm{P}<0.05,{ }^{\star \star \star}$ for $\mathrm{P}<0.001$.

first-round PCR temperature regimen. PCR products were purified using the QIAquick PCR Purification Kit (Qiagen, Germany). Illumina adaptors were ligated using NEBNext ${ }^{\circledR}$ Ultra $^{\mathrm{TM}}$ II DNA Library Prep Kit for Illumina ${ }^{\circledR}$ (New England Biolabs, USA) according to the manufacturer's protocol and sequencing in the Illumina NovaSeq 6000 platform with the PE150 mode. The parameters of sequencing quality were shown in (Supplementary Table 7).

\section{Data Processing and Analysis}

The original data obtained from high-throughput sequencing were converted to raw sequence reads by base calling, and the results were stored in FASTQ format. Low-quality reads and reads without primers were discarded. PCR and sequencing errors were corrected by unique molecular identifiers (UMIs). The reads with same UMI were one clone. Only duplicate reads with different UMIs will be kept in downstream processing. A clonotype was defined by the CDR3 amino acid sequence for further analysis (the same UMI reads must be more than two). TCR $\beta \mathrm{V}, \mathrm{D}$, and J genes and clonotype were defined according to IMGT59 and IgBLAST; TCR $\beta$ VDJ combination was defined by MiXCR. We performed mRNA-Seq profiling on 70 samples. Reads were mapped to the IPD-IMGT/HLA database and HLAHD determining HLA alleles using mRNA-seq results (47).

\section{Statistical Analysis}

Categorical variables were described as count (\%), and a twogroup comparison was performed using Fisher's exact test. Continuous variables were expressed as median and interquartile range (IQR) values and compared by the MannWhitney $U$ test between groups. The Spearman correlation coefficient was calculated to assess the association between two vectors of quantitative variables. The significance of monotonic trend across multiple groups was assessed by Cuzick's test (35). The significance of overlap between two gene sets was assessed by hypergeometric test (the "dhyper" function of R software). All statistical analyses were performed using $\mathrm{R}$ software (version 4.0.2). A two-sided p-value lower than 0.05 was considered as statistically significant.

\section{DATA AVAILABILITY STATEMENT}

The raw data supporting the conclusions of this article will be made available by the authors, without undue reservation.

\section{ETHICS STATEMENT}

This study was approved by the ethics committees of the above four participant hospitals. Written informed consent was obtained from all participants enrolled in this study.

\section{AUTHOR CONTRIBUTIONS}

JX, KW, and DW conceived and supervised the project. YS, DL, JL, and WH provided COVID-19 blood samples and clinical information. YuL, JH, DL, YW, and JZ performed the experiments and conducted the sample preparation. KW, YiL. JH, YW, YuL, and WF performed the data analysis. $\mathrm{HH}, \mathrm{WW}$, and LL coordinated the projects. YiL, JH, and KW wrote the manuscript. All authors contributed to the article and approved the submitted version.

\section{FUNDING}

This study was funded by the Science and Technology Innovation Project of Foshan Municipality (2020001000431), National Key Research and Development Project (2020YFA0708001), and Guangdong Provincial Science and Technology Project (2020A1414010363).

\section{SUPPLEMENTARY MATERIAL}

The Supplementary Material for this article can be found online at: https://www.frontiersin.org/articles/10.3389/fimmu.2021. 769442/full\#supplementary-material 


\section{REFERENCES}

1. Wu Z, McGoogan JM. Characteristics of and Important Lessons From the Coronavirus Disease 2019 (COVID-19) Outbreak in China: Summary of a Report of 72314 Cases From the Chinese Center for Disease Control and Prevention. JAMA (2020) 323(13):1239-42. doi: 10.1001/jama.2020.2648

2. Guan W-j, Liang W-h, Zhao Y, Liang H-r, Chen Z-s, Li Y-m, et al. Comorbidity and Its Impact on 1590 Patients With COVID-19 in China: A Nationwide Analysis. Eur Respir J (2020) 55(5):2000547. doi: 10.1183/ 13993003.00547-2020

3. Wang D, Hu B, Hu C, Zhu F, Liu X, Zhang J, et al. Clinical Characteristics of 138 Hospitalized Patients With 2019 Novel Coronavirus-Infected Pneumonia in Wuhan, China. JAMA (2020) 323(11):1061-9. doi: 10.1001/jama.2020.1585

4. Lan L, Xu D, Ye G, Xia C, Wang S, Li Y, et al. Positive RT-PCR Test Results in Patients Recovered From COVID-19. JAMA (2020) 323(15):1502-3. doi: 10.1001/jama.2020.2783

5. Lucas C, Wong P, Klein J, Castro TBR, Silva J, Sundaram M, et al. Longitudinal Analyses Reveal Immunological Misfiring in Severe COVID19. Nature (2020) 584(7821):463-9. doi: 10.1038/s41586-020-2588-y

6. Mathew D, Giles JR, Baxter AE, Oldridge DA, Greenplate AR, Wu JE, et al. Deep Immune Profiling of COVID-19 Patients Reveals Distinct Immunotypes With Therapeutic Implications. Science (2020) 369(6508): eabc8511. doi: 10.1126/science.abc8511

7. Zhang JY, Wang XM, Xing X, Xu Z, Zhang C, Song JW, et al. Single-Cell Landscape of Immunological Responses in Patients With COVID-19. Nat Immunol (2020) 21(9):1107-18. doi: 10.1038/s41590-020-0762-x

8. Giamarellos-Bourboulis EJ, Netea MG, Rovina N, Akinosoglou K, Antoniadou A, Antonakos N, et al. Complex Immune Dysregulation in COVID-19 Patients With Severe Respiratory Failure. Cell Host Microbe (2020) 27(6):992-1000.e3. doi: 10.1016/j.chom.2020.04.009

9. Song JW, Zhang C, Fan X, Meng FP, Xu Z, Xia P, et al. Immunological and Inflammatory Profiles in Mild and Severe Cases of COVID-19. Nat Commun (2020) 11(1):3410. doi: 10.1038/s41467-020-17240-2

10. Wilk AJ, Rustagi A, Zhao NQ, Roque J, Martínez-Colón GJ, McKechnie JL, et al. A Single-Cell Atlas of the Peripheral Immune Response in Patients With Severe COVID-19. Nat Med (2020) 26(7):1070-6. doi: 10.1038/s41591-0200944-y

11. Qin C, Zhou L, Hu Z, Zhang S, Yang S, Tao Y, et al. Dysregulation of Immune Response in Patients With Coronavirus 2019 (COVID-19) in Wuhan, China. Clin Infect Dis (2020) 71(15):762-8. doi: 10.1093/cid/ciaa248

12. Wen W, Su W, Tang H, Le W, Zhang X, Zheng Y, et al. Immune Cell Profiling of COVID-19 Patients in the Recovery Stage by Single-Cell Sequencing. Cell Discov (2020) 6:31. doi: 10.1038/s41421-020-0168-9

13. Long Q-X, Tang X-J, Shi Q-L, Li Q, Deng H-J, Yuan J, et al. Clinical and Immunological Assessment of Asymptomatic SARS-CoV-2 Infections. Nat Med (2020) 26(8):1200-4. doi: 10.1038/s41591-020-0965-6

14. An J, Liao X, Xiao T, Qian S, Yuan J, Ye H, et al. Clinical Characteristics of the Recovered COVID-19 Patients With Re-Detectable Positive RNA Test. medRxiv (2020) 8(17):1084. doi: 10.1101/2020.03.26.20044222

15. Rothe C, Schunk M, Sothmann P, Bretzel G, Froeschl G, Wallrauch C, et al. Transmission of 2019-Ncov Infection From an Asymptomatic Contact in Germany. N Engl J Med (2020) 382(10):970-1. doi: 10.1056/NEJMc2001468

16. Bai Y, Yao L, Wei T, Tian F, Jin D-Y, Chen L, et al. Presumed Asymptomatic Carrier Transmission of COVID-19. JAMA (2020) 323(14):1406-7. doi: 10.1001/jama.2020.2565

17. Zhu H, Fu L, Jin Y, Shao J, Zhang S, Zheng N, et al. Clinical Features of COVID-19 Convalescent Patients With Re-Positive Nucleic Acid Detection. J Clin Lab Anal (2020) 34(7):e23392. doi: 10.1002/jcla.23392

18. Gao Z, Xu Y, Guo Y, Xu D, Zhang L, Wang X, et al. A Systematic Review of Re-Detectable Positive Virus Nucleic Acid Among COVID-19 Patients in Recovery Phase. Infect Genet Evol: J Mol Epidemiol Evol Genet Infect Dis (2020) 85:104494. doi: 10.1016/j.meegid.2020.104494

19. Zhang J, Lin D, Li K, Ding X, Li L, Liu Y, et al. Transcriptome Analysis of Peripheral Blood Mononuclear Cells Reveals Distinct Immune Response in Asymptomatic and Re-Detectable Positive COVID-19 Patients. Front Immunol (2021) 12:716075. doi: 10.3389/fimmu.2021.716075

20. Ranasinghe S, Lamothe PA, Soghoian DZ, Kazer SW, Cole MB, Shalek AK, et al. Antiviral CD8(+) T Cells Restricted by Human Leukocyte Antigen Class
II Exist During Natural HIV Infection and Exhibit Clonal Expansion. Immunity (2016) 45(4):917-30. doi: 10.1016/j.immuni.2016.09.015

21. Song I, Gil A, Mishra R, Ghersi D, Selin LK, Stern LJ. Broad TCR Repertoire and Diverse Structural Solutions for Recognition of an Immunodominant CD8(+) T Cell Epitope. Nat Struct Mol Biol (2017) 24(4):395-406. doi: $10.1038 / \mathrm{nsmb} .3383$

22. Emerson RO, DeWitt WS, Vignali M, Gravley J, Hu JK, Osborne EJ, et al. Immunosequencing Identifies Signatures of Cytomegalovirus Exposure History and HLA-Mediated Effects on the T Cell Repertoire. Nat Genet (2017) 49(5):659-65. doi: 10.1038/ng.3822

23. Soon CF, Behrendt P, Todt D, Manns MP, Wedemeyer H, Sällberg Chen M, et al. Defining Virus-Specific CD8+ TCR Repertoires for Therapeutic Regeneration of T Cells Against Chronic Hepatitis E. J Hepatol (2019) 71 (4):673-84. doi: 10.1016/j.jhep.2019.06.005

24. Wendel BS, del Alcazar D, He C, Del Río-Estrada PM, Aiamkitsumrit B, Ablanedo-Terrazas Y, et al. The Receptor Repertoire and Functional Profile of Follicular T Cells in HIV-Infected Lymph Nodes. Sci Immunol (2018) 3(22): eaan8884. doi: 10.1126/sciimmunol.aan8884

25. Kuri-Cervantes L, Pampena MB, Meng W, Rosenfeld AM, Betts MR. Comprehensive Mapping of Immune Perturbations Associated With Severe COVID-19. Sci Immunol (2020) 5(49):eabd7114. doi: 10.1126/sciimmunol.abd7114

26. Wauters E, Van Mol P, Garg AD, Jansen S, Van Herck Y, Vanderbeke L, et al. Discriminating Mild From Critical COVID-19 by Innate and Adaptive Immune Single-Cell Profiling of Bronchoalveolar Lavages. Cell Res (2021) 31(3):272-90. doi: 10.1038/s41422-020-00455-9

27. Schultheiß C, Paschold L, Simnica D, Mohme M, Willscher E, von Wenserski L, et al. Next-Generation Sequencing of $\mathrm{T}$ and B Cell Receptor Repertoires From COVID-19 Patients Showed Signatures Associated With Severity of Disease. Immunity (2020) 53(2):442-55. doi: 10.1016/j.immuni.2020.06.024

28. Galson JD, Schaetzle S, Bashford-Rogers RJM, Raybould MIJ, Kovaltsuk A, Kilpatrick GJ, et al. Deep Sequencing of B Cell Receptor Repertoires From COVID-19 Patients Reveals Strong Convergent Immune Signatures. Front Immunol (2020) 11:605170. doi: 10.3389/fimmu.2020.605170

29. Niu X, Li S, Li P, Pan W, Wang Q, Feng Y, et al. Longitudinal Analysis of T and B Cell Receptor Repertoire Transcripts Reveal Dynamic Immune Response in COVID-19 Patients. Front Immunol (2020) 11:582010. doi: 10.3389/fimmu.2020.582010

30. Zhou Y, Zhang J, Wang D, Wang D, Guan W, Qin J, et al. Profiling of the Immune Repertoire in COVID-19 Patients With Mild, Severe, Convalescent, or Retesting-Positive Status. J Autoimmun (2021) 118:102596. doi: 10.1016/ j.jaut.2021.102596

31. Woodruff MC, Ramonell RP, Nguyen DC, Cashman KS, Saini AS, Haddad NS, et al. Extrafollicular B Cell Responses Correlate With Neutralizing Antibodies and Morbidity in COVID-19. Nat Immunol (2020) 21(12):150616. doi: 10.1038/s41590-020-00814-z

32. Xiong F, Tang H, Liu L, Tu C, Tian JB, Lei CT, et al. Clinical Characteristics of and Medical Interventions for COVID-19 in Hemodialysis Patients in Wuhan, China. J Am Soc Nephrol (2020) 31(7):1387-97. doi: 10.1681/asn.2020030354

33. Goldrath AW, Bevan MJ. Selecting and Maintaining a Diverse T-Cell Repertoire. Nature (1999) 402(6759):255-62. doi: 10.1038/46218

34. Liu X, Zhang W, Zhao M, Fu L, Liu L, Wu J, et al. T Cell Receptor $\beta$ Repertoires as Novel Diagnostic Markers for Systemic Lupus Erythematosus and Rheumatoid Arthritis. Ann Rheum Dis (2019) 78(8):1070-. doi: 10.1136/ annrheumdis-2019-215442

35. Cuzick JA. Wilcoxon-Type Test for Trend. Stat Med (1985) 4(1):87-90. doi: $10.1002 / \mathrm{sim} .4780040112$

36. Lee Y-H, Hong CM, Kim DH, Lee TH, Lee J. Clinical Course of Asymptomatic and Mildly Symptomatic Patients With Coronavirus Disease Admitted to Community Treatment Centers, South Korea. Emerg Infect Dis (2020) 26 (10):2346-52. doi: 10.3201/eid2610.201620

37. Lee S, Kim T, Lee E, Lee C, Kim H, Rhee H, et al. Clinical Course and Molecular Viral Shedding Among Asymptomatic and Symptomatic Patients With SARS-CoV-2 Infection in a Community Treatment Center in the Republic of Korea. JAMA Intern Med (2020) 180(11):1447-52. doi: 10.1001/ jamainternmed.2020.3862

38. Pan Y, Yu X, Du X, Li Q, Li X, Qin T, et al. Epidemiological and Clinical Characteristics of 26 Asymptomatic Severe Acute Respiratory Syndrome Coronavirus 2 Carriers. J Infect Dis (2020) 221(12):1940-7. doi: 10.1093/infdis/jiaa205 
39. Oh H-LJ, Chia A, Chang CXL, Leong HN, Ling KL, Grotenbreg GM, et al. Engineering T Cells Specific for a Dominant Severe Acute Respiratory Syndrome Coronavirus CD8 T Cell Epitope. J Virol (2011) 85(20):1046471. doi: 10.1128/JVI.05039-11

40. Le Bert N, Tan AT, Kunasegaran K, Tham CYL, Hafezi M, Chia A, et al. SARS-CoV2-Specific T Cell Immunity in Cases of COVID-19 and SARS, and Uninfected Controls. Nature (2020) 584(7821):457-62. doi: 10.1038/s41586-020-2550-Z

41. Snyder TM, Gittelman RM, Klinger M, May DH, Osborne EJ, Taniguchi R, et al. Magnitude and Dynamics of the T-Cell Response to SARS-CoV-2 Infection at Both Individual and Population Levels. medRxiv (2020), 2020.07.31.20165647. doi: 10.1101/2020.07.31.20165647

42. Nolan S, Vignali M, Klinger M, Dines JN, Kaplan IM, Svejnoha E, et al. A Large-Scale Database of T-Cell Receptor Beta $(\operatorname{Tcr} \beta)$ Sequences and Binding Associations From Natural and Synthetic Exposure to SARS-CoV-2. Res Square (2020) rs.3.rs-51964. doi: 10.21203/rs.3.rs-51964/v1

43. National Health Commission of the People's Republic of China. Protocol on Prevention and Control of Novel Coronavirus Pneumonia (Edition 6). (2020).

44. National Health Commission of the People's Republic of China. Diagnosis and Treatment Protocol for Novel Coronavirus Pneumonia (Trial Version 7). (2020).

45. Mamedov IZ, Britanova OV, Zvyagin IV, Turchaninova MA, Bolotin DA, Putintseva EV, et al. Preparing Unbiased T-Cell Receptor and Antibody cDNA Libraries for the Deep Next Generation Sequencing Profiling. Front Immunol (2013) 4:456. doi: 10.3389/fimmu.2013.00456

46. Chen Y, Xu Y, Zhao M, Liu Y, Gong M, Xie C, et al. High-Throughput T Cell Receptor Sequencing Reveals Distinct Repertoires Between Tumor and
Adjacent non-Tumor Tissues in HBV-Associated HCC. Oncoimmunology (2016) 5(10):e1219010. doi: 10.1080/2162402x.2016.1219010

47. Kawaguchi S, Higasa K, Shimizu M, Yamada R, Matsuda F. HLA-HD: An Accurate HLA Typing Algorithm for Next-Generation Sequencing Data. Hum Mutat (2017) 38(7):788-97. doi: 10.1002/humu.23230

Conflict of Interest: Authors YW, WF and LL were employed by company Guangzhou Huayin Medical Laboratory Center Ltd.

The remaining authors declare that the research was conducted in the absence of any commercial or financial relationships that could be construed as a potential conflict of interest.

Publisher's Note: All claims expressed in this article are solely those of the authors and do not necessarily represent those of their affiliated organizations, or those of the publisher, the editors and the reviewers. Any product that may be evaluated in this article, or claim that may be made by its manufacturer, is not guaranteed or endorsed by the publisher.

Copyright (C) $2022 \mathrm{Li}, \mathrm{Hu}$, Wang, Liu, Shi, Zhang, Liu, Lin, Lin, Hu, He, Wang, Fan, $L i$, Wang, Wang and Xu. This is an open-access article distributed under the terms of the Creative Commons Attribution License (CC BY). The use, distribution or reproduction in other forums is permitted, provided the original author(s) and the copyright owner(s) are credited and that the original publication in this journal is cited, in accordance with accepted academic practice. No use, distribution or reproduction is permitted which does not comply with these terms. 